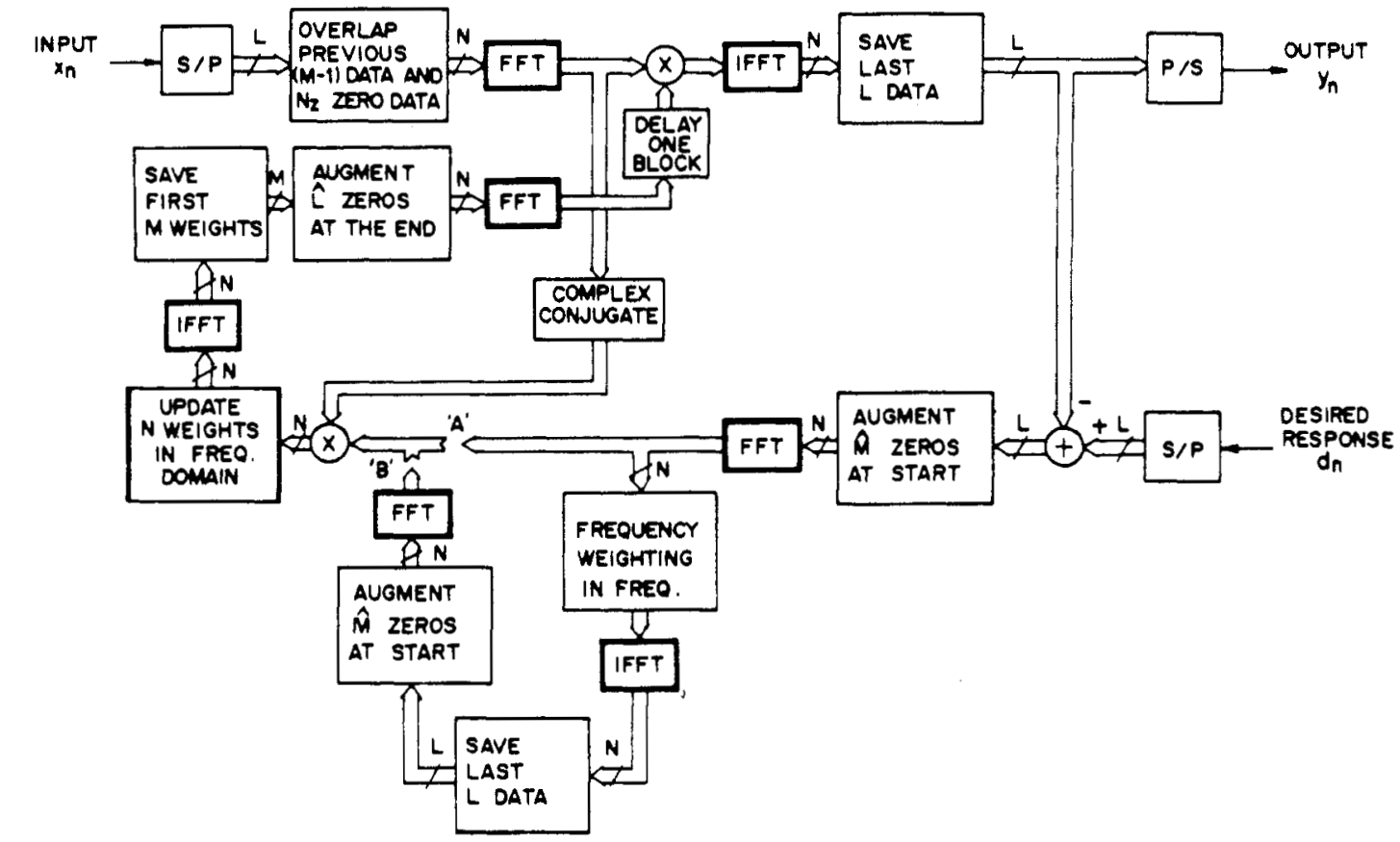

Fig. 1. Realization of FBLMS and FWBLMS ADF's using FFT and overlap-save sectioning procedure $\left(N=L+M-1+N_{z}, i \triangleq L-1+N_{z}, \dot{N} \triangleq N_{1}-1+N_{z}\right.$, and $\left.N_{z} \geqslant 0\right)$. (Note: FBLMS ADF is realized with the position " $A$ " connected and FWBLMS ADF is realized with the position " $B$ " connected. $N_{z}$ is the number of zero data needed for augmenting the input data, thereby allowing to choose a suitable transform of length $N \quad S / P=$ serial-to-parallel conversion and $P / S=$ parallel-to-serial conversion.]

\section{A Frequency-Weighted Block LMS Algorithm and Its Application to Speech Processing}

\section{C. LEE, C. K. UN, AND D. H. CHO}

In this letter, we propose a frequency-weighted block LMS (FWBLMS) algorithm that is based on minimization of the trequency-weighted block mean-squared error. The FWBLMS al. gorithm has an important advantage over the time-domain LMIS algorithm in that it can be designed with different frequency weighting on error signal depending on the relative significance of various frequency bands. Application of the FWBLMS algorithm in adaptive linear prediction of speech is discussed.

\section{INTRODUCION}

Recently, considerable research effort has been directed towards efficient realization of adaptive digital filters (ADFs) using the block least mean square (BLMS) algorithms [1]-[4] In the BLMS ADFs, filter weights are updated once in every block rather than at every sampling instant. Since the block-estimated gradient becomes cross-correlation between the input and the error signal in the block, the BLMS ADF can be realized efficiently using the fast Fourier transform (FFT). The frequency-domain BLMS (FBLMS) ADF in which filter weights are adjusted in the frequency domain converges fast even for a highly correlated input [1], [2]. In this letter we investigate another attractive feature of the FBLMS ADF. Specifically, we study a frequency-weighted BLMS (FWBLMS) weight adjustment algorithm that is based on minimizing a weighted sum of frequency-domain errors, and discuss the use of the algorithm in linear prediction of speech for improving the subjective quality of speech.

Manuscript received fuly 30, 1984; revised October 23, 1984

The authors are with the Communications Research Laboratory, De. partment of Electrical Engineering, Korea Advanced Institute of Science and Technology, Seoul, Korea.

\section{A FREQUENCY-WEICHTED BLMS ALCORITHM}

Let us consider a transversal ADF operated on the block-by-block basis. Prior to derivation of the FWBLMS algorithm, we briefly review the FBLMS ADF. Let $M, L$, and $N$ be the number of filter weights, the block length, and the transform length of FFT, respectively. The FBLMS ADF shown in Fig. 1 can be obtained by minimizing the frequency-domain block mean-squared error (BMSE). In this $A D F$, the frequency-domain error vector $e_{k}$ in the $k$ th block is given by [1]

$$
e_{k}=d_{k}-\mathscr{P}_{0 . L}\left(X_{k} \mathscr{P}_{N .0} w_{k}\right)
$$

where $\boldsymbol{d}_{k}$ and $\boldsymbol{w}_{k}$ are the $(N \times 1)$ desired response and filter weight vectors, respectively, both in the frequency domain, and $x_{k}$ is an $(N \times N)$ diagonal matrix whose diagonal elements are the transformed input data [see Fig. 1]. In (1), the two $(N \times N)$ matrices $\mathscr{F}_{0 . t}$ and $\mathscr{P}_{\text {ito }}$ realize the sectioning procedures needed for computing the filter output and adjusting the filter weights, respectively. They are defined as

and

$$
\mathscr{P}_{0 . L} \triangleq F\left[\begin{array}{ll}
0 & 0 \\
0 & l_{L}
\end{array}\right] F^{-1}
$$

$$
\mathscr{P}_{M, O} \stackrel{\triangleq}{=}\left[\begin{array}{ll}
N_{N 1} & 0 \\
0 & 0
\end{array}\right] F^{-1}
$$

where $F$ is the $(N \times N)$ discrete Fourier transtorm matrix, $I_{i}$ and $I_{L}$ denote $(L \times L)$ and $(M \times M)$ identity matrices, respectively, and 0 is a zero matrix.

As a performance criterion in adjusting the filter weights, the frequency-weighted BMSE (FWBNSE) $\varepsilon^{\prime w}$ is defined by

$$
\varepsilon^{i w} \triangleq E\left[e_{k}^{*} \Gamma e_{k}\right]
$$

where the asterisk and $E[\cdot]$ denote complex-conjugate transpose of a matrix and statistical expectation, respectively. In (3), $\Gamma$ is an $(N \times N)$ diagonal matrix whose diagonal elements are of nonnegative values and their relative magnitudes represent the relative 
significance of each frequency component. Following the same approach used for the FBLMS ADF [2], we can have from (1) and (3) a gradient of the FWBMSE with respect to $w_{k}$ as [5]

$$
\nabla \varepsilon^{f w}\left(w_{k}\right) \triangleq \frac{\partial \varepsilon^{f w}}{\partial w_{k}}=-2 E\left[\mathscr{P}_{M, O} \boldsymbol{X}_{k}^{*} \mathscr{P}_{0, L} \Gamma e_{k}\right]
$$

Thus, using an instantaneously estimated gradient, we obtain from (4) an FWBLMS weight adjustment algorithm as the following:

$$
w_{k+1}=w_{k}+\mu \mathscr{P}_{M, O} X_{k}^{*} \mathscr{P}_{0, \iota} \Gamma e_{k}
$$

where $\mu$ is a convergence factor controlling the convergence behavior of the algorithm. When the initial values of the filter weights are zero, the algorithm of (5) can be realized alternatively as [1], [5]

$$
w_{k+1}=\mathscr{P}_{M, 0}\left(w_{k}+\mu x_{k}^{*} \mathscr{P}_{0, L} \Gamma e_{k}\right) \text {. }
$$

In Fig. 1, a block diagram of the FWBLMS ADF using the algorithm of (6) is shown together with that of the FBLMS ADF. It is noted here that, when $\Gamma$ is an identity matrix, the FWBLMS algorithm becomes identical to the FBLMS algorithm since $\mathscr{P}_{0, L} \boldsymbol{e}_{k}=\boldsymbol{e}_{k}$. Also, it is noted that, when $L$ is sufficiently larger than $M, \mathscr{P}_{0}$, can be approximated as an identity matrix. In that case, one can eliminate the FFT and inverse FFT operations that are needed just after the frequency weighting operation in the FWBLMS ADF [see (6) and Fig. 1].

\section{Application of the FWBLMS ALGORITHM}

It is known that some frequency weighting on the error signal in a speech processing system is desirable in improving the subjective quality of speech [6]. One of the commonly accepted aspects of speech perception is that noise in the frequency range of 1 to $3 \mathrm{kHz}$ introduces more serious degradation in intelligibility than that in other range [7].

To investigate the effect of frequency weighting, we have studied adaptive linear prediction of speech using the FWBLMS algorithm by computer simulation, in which we have used a one-step predictor with ten prediction coefficients. The real speech input having unity signal power was sampled at $8 \mathrm{kHz}$ and processed at every $22.5 \mathrm{~ms}$. For frequency weighting we used a $C$-message weighting function [8] that emphasizes the error signal in the frequency band of 1 to $3 \mathrm{kHz}$. In Fig. 2, the spectral envelopes of the Hammingwindowed prediction errors of a voiced speech block are compared

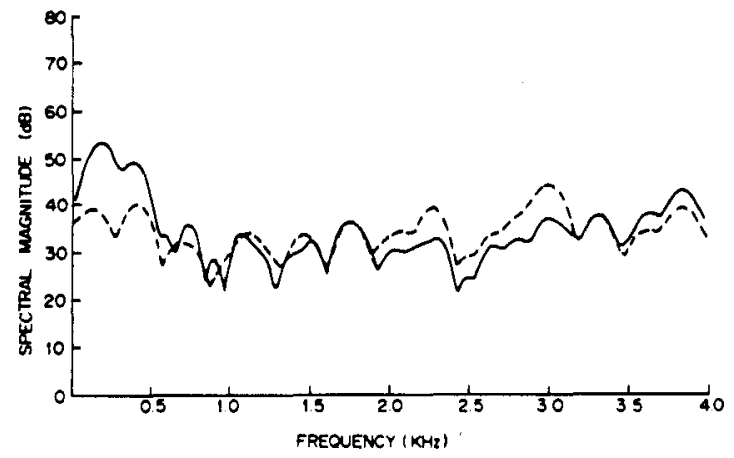

Fig. 2. Spectral envelopes of prediction errors for a voiced speech block with (solid line) and without (dotted line) C-message frequency weighting

for the cases with and without frequency weighting. It is noted that our simulation results for the cases with and without the $\mathscr{P}_{0 . t}$ operation after frequency weighting do not show appreciable differences in the performance of our application example with $M=$ 10 and $L=180$. It is clearly seen from Fig. 2 that, by having more emphasis in the range of 1 to $3 \mathrm{kHz}$, the frequency-domain error in that range can be reduced at the expense in other frequency region. According to our preliminary results of computer simulation, one can improve the performance of the FWBLMS adaptive linear pre- dictor by more than $2 \mathrm{~dB}$ in the frequency-weighted signal-to-noise ratio.

REFERENCES

[1] D. Mansour and A. H. Gray, Jr., "Performance characteristics of the unconstrained frequency-domain adaptive filter," in Proc. 1982 IEEE int. Symp. on Circuits and Systems (May 1982), pp. 695-698.

[2] - "Unconstrained frequency-domain adaptive filter," IEEE Trans. Acoust., Speech, Signal Process., vol. ASSP-30, pp. 726-734, Oct 1982.

[3] G. A. Clark, S. R. Parker, and S. K. Mitra, "A unified approach to time- and frequency-domain realization of FIR adaptive digital filters," IEEE Trans. Acoust., Speech, Signal Process., vol. ASSP-31, pp. 1073-1083, Oct 1983.

[4] J. C. Lee and C. K. Un, "A reduced structure of the frequencydomain block LMS adaptive digital filter," Proc. IEEE, vol. 72, no. 12 , pp. 1816-1818, Dec. 1984.

[5] _ "Performances of time- and frequency-domain block LMS adaptive digital filters," to be submitted for publication in IEEE Trans. Acoust., Speech, Signal Process.

[6] J. 5. Lim, Ed., Speech Enhancement. Englewood Cliffs, NJ: Pren tice-Hall, 1983.

[7] 5. C. Wilson and 5. Husain, "Adaptive tree encoding of speech at 8000 bits $/ 5$ with a frequency-weighted error criterion," IEEE Trans. Commun., vol. COM-27, pp. 165-170, Jan. 1979.

[8] A. J. Aikens and D. A. Lewinski, "Evaluation of message circuit noise," Bell Syst. Tech. J., vol. 39, pp. 879-910, July 1960.

\section{The Observation of Individual \\ Natural-Frequency Resonances of Radar Targets Through the Scattering of Long Pulses}

\section{W. E. HOWELL AND H. ÜBERALL}

The Singularity Expansion Method has shown the transient echo amplitude of radar targets to be characterized by complex-frequency poles and their residues. We note that the use of pulsed signals of long duration permits identification of individual pole resonances by the observation of their ringing

The Singularity Expansion Method (SEM) [1] is based on the observation that the echoes of radar pulses scattered from a finite target appear as the superposition of damped sinusoids,

$$
f_{\mathrm{sc}}(t)=\sum_{\alpha=1}^{N} R_{\alpha} e^{s_{a} t}
$$

with complex amplitudes $R_{\alpha}$ and exponents $\hat{S}_{\alpha}$. Such a signal shape indicates the presence of complex-frequency poles in the scattering amplitude, via the Laplace transform

$$
f_{s c}(s)=\sum_{\alpha=1}^{N} \frac{R_{\alpha}}{s-\hat{s}_{\alpha}}
$$

where $s=\omega / i$, and $\omega_{\alpha}=\operatorname{Im} \hat{s}_{\alpha}$ are the natural frequencies of the target whose existence gives rise to resonances in the scattering amplitude of (1b).

This latter equation corresponds to a single-frequency excitation of the resonances. If the incident amplitude is pulsed in time, its Fourier spectrum $C(k)$ weighs the factor $R_{\alpha}$ in (1b), where $k=\omega / c$

A short pulse is characterized by a wide spectrum $C(k)$; for $G(k) \equiv 1$, one has $f_{\text {inc }}(z, t)=\delta(z-c t)$. In such a case, many pole terms contribute to (1b).

An incident pulse of long duration has a narrow spectrum $C(k)$ whose weight in (1b) can radically limit the number of poles contributing to (1). In fact, if the pulse duration is chosen long

Manuscript received fune 14, 1984; revised October 17, 1984. Portions of this work were supported by the Naval Research Laboratory.

The authors are with the Department of Physics, The Catholic University of America. Washington, DC 20064, USA 